\title{
Diffusion Coefficient of a Brownian Particle with a Friction Function Given by a Power Law
}

\author{
Benjamin Lindner
}

Received: 6 June 2007 / Accepted: 13 September 2007 / Published online: 25 October 2007

(C) Springer Science+Business Media, LLC 2007

\begin{abstract}
Nonequilibrium biological systems like moving cells or bacteria have been phenomenologically described by Langevin equations of Brownian motion in which the friction function depends on the particle's velocity in a nonlinear way. An important subclass of such friction functions is given by power laws, i.e., instead of the Stokes friction constant $\gamma_{0}$ one includes a function $\gamma(v) \sim v^{2 \alpha}$. Here I show using a recent analytical result as well as a dimension analysis that the diffusion coefficient is proportional to a simple power of the noise intensity $D$ like $D^{(1-\alpha) /(1+\alpha)}$ (independent of spatial dimension). In particular the diffusion coefficient does not depend on the noise intensity at all, if $\alpha=1$, i.e., for a cubic friction $F_{\text {fric }}=-\gamma(v) v \sim v^{3}$. The exact prefactor is given in the one-dimensional case and a fit formula is proposed for the multi-dimensional problem. All results are confirmed by stochastic simulations of the system for $\alpha=1,2$, and 3 and spatial dimension $d=1,2$, and 3. Conclusions are drawn about the strong noise behavior of certain models of self-propelled motion in biology.
\end{abstract}

Keywords Diffusion · Langevin equation · Nonlinear friction

\section{Introduction}

To the Scottish botanist Robert Brown the motion of small suspended particles in liquid looked rather agitated and lively at the first glance [1]. As is well known, he proved with great patience that the origin of the motion was not organic; however, he was unable to tell what its cause was. Much later Einstein [2] showed that the origin of the so-called Brownian motion lays in the kicks of the surrounding molecules of the liquid and that one has to expect such a random motion of the Brownian (i.e., micrometer-sized) particles, if one believes in statistical mechanics and in the particle nature of matter.

Paul Langevin [3] gave the first example for a stochastic differential equation to describe this motion in a particularly elegant and simple way. The Langevin equation is

B. Lindner $(\bowtie)$

Max-Planck-Institut für Physik komplexer Systeme, Nöthnitzer Str. 38, 01187 Dresden, Germany

e-mail: benji@pks.mpg.de 
Newton's law in $d$ dimension with a Stokes friction force (proportional to velocity) and a stochastic noise that describes the random kicks of the surrounding water molecules:

$$
\dot{\boldsymbol{x}}=\boldsymbol{v}, \quad m \dot{\boldsymbol{v}}=-\gamma \boldsymbol{v}+\sqrt{2 D} \boldsymbol{\xi}(t) .
$$

Here $m, \gamma$, and $D$ are the particle mass, the friction coefficient, and the noise intensity, respectively. Since the Brownian particle is in equilibrium with the liquid the noise intensity is related to temperature and friction coefficient by $D=\gamma k_{B} T$ ( $k_{B}$ is Boltzmann's constant).

Ironically, within the last two decades generalizations of the very same Langevin equation have been used to describe the motion of living objects (molecular motors, crawling cells, flocks of animals) that are definitely out of thermodynamic equilibrium (see, for instance, refs.[4-8]). Instead of eq. (1) one considers

$$
\dot{\boldsymbol{x}}=\boldsymbol{v}, \quad m \dot{\boldsymbol{v}}=-\hat{\gamma}(v) \boldsymbol{v}+\sqrt{2 D} \boldsymbol{\xi}(t)
$$

where the friction is now an even function of the speed $v$. The speed in the $d$-dimensional case is given by

$$
v=\sqrt{\sum_{j=1}^{d} v_{j}^{2}} .
$$

A typical choice for the nonlinear friction function is $\hat{\gamma}(v)=v^{2}-u_{0}^{2}$ (Rayleigh friction) which for $|v|<u_{0}$ becomes negative, i.e., here the friction acts as an energy pump. In general, one may approximate any even friction function by a power series of even powers of $v$

$$
\hat{\gamma}(v)=\sum_{n=0}^{\alpha} \gamma_{n} v^{2 n} .
$$

In particular, for the strong noise behavior only the highest power matters. Note, that the asymptotics at large speed may also be linear (and thus we would have to take $\alpha=0$ in the above formula) even if the function is nonlinear for small velocities (see the so-called simplified depot model as an example [4, 9]). It is thus interesting to study friction functions that follow a simple power law.

In this paper I will study the diffusion coefficient for a d-dimensional system with a friction function

$$
\hat{\gamma}(v) \sim v^{2 \alpha},
$$

i.e., I deal with a Langevin equation like

$$
m \dot{\boldsymbol{v}}=-\gamma v^{2 \alpha} \boldsymbol{v}+\sqrt{2 D} \boldsymbol{\xi}(t)
$$

where I dropped the index of the prefactor $\left(\gamma_{\alpha}=\gamma\right)$. I aim at determining the diffusion coefficient

$$
D_{\text {eff }}=\lim _{t \rightarrow \infty} \frac{1}{2 t d} \sum_{j=1}^{d}\left\langle\left[x_{j}(t)-x_{j}(0)\right]^{2}\right\rangle .
$$

There is another motivation to study this coefficient: Simple nonlinear stochastic differential equations like (6) can be used to generate colored noise with non-Gaussian statistics (for a review on problems where colored-noise driving is an issue, see [10]). According to the 
Kubo theorem the diffusion coefficient is given by the integrated correlation function of the velocity

$$
D_{\text {eff }}=\frac{1}{d} \int_{0}^{\infty} d \tau\langle\boldsymbol{v}(t) \boldsymbol{v}(t+\tau)\rangle
$$

In particular, in the one-dimensional case, the diffusion coefficient corresponds to the noise intensity of the velocity-not to be confused with the noise intensity $D$ of the driving white noise. If the dynamics given in (6) is regarded as a noise generator, one would like to know the basic characteristics of this noise: its intensity (given by $D_{\text {eff }}$ ) and its correlation time and if possible the dependence of these parameters on the system's parameters $\gamma$ and $D$. What will be discussed in this paper may be useful for designing more complex colored-noise generators than just the Ornstein-Uhlenbeck process and the randomtelegraph noise commonly used. As shown in the following, for the dynamics equation (6) holds

$$
D_{\text {eff }} \sim D^{\beta} \gamma^{\beta^{\prime}} m^{\beta^{\prime \prime}}
$$

In this work I will solely focus on a discussion of the dependence on the noise intensity $D$.

In the one-dimensional case, one can also calculate the factor of proportionality by means of an exact quadrature result recently derived [11]. I will demonstrate the scaling relations for the cases $\alpha=0$ (normal Brownian motion) and $\alpha=1$ (the high-speed limit of the Rayleigh friction), as well as for two examples of even stronger nonlinearity with $\alpha=2$ and $\alpha=3$. I will explain by intuitive arguments the remarkable findings that the diffusion coefficient for $\alpha=1$ does not depend on the noise intensity at all and that it decreases with increasing noise for $\alpha>1$.

It is maybe worth to stress that a Langevin system with a nonlinear friction equation (5) (with $\alpha>0$ ) and constant noise can hardly model an equilibrium system since dissipation and fluctuations have obviously different origins - one depends on the particle speed, the other does not. One can, however, construct an equilibrium model where both friction and noise intensity depend in a similar way on the speed (governed by a generalized Einstein relation). I will briefly discuss why in this case the diffusion coefficient must diverge. For further studies of equilibrium models with nonlinear friction I refer the interested reader to Refs. [11, 12].

This paper is structured as follows. In the next section, I use the exact result for the diffusion coefficient in the one-dimensional case given in [11] to derive the scaling relation (9), the exponents, and in this simple case also the proportionality constant. The more general $d$ dimensional case is treated in Sect. 3, showing that the value of the exponent $\beta$ does not change as one considers higher spatial dimension. In Sect. 4 the analytical findings will be compared to numerical simulations and an explanation for the independence of diffusion of noise intensity for the special case $\alpha=1$ is given. In Sect. 5 two extensions of the model (deviations from a pure power law and an equilibrium version of a system with pure power law friction) will be discussed. In Sect. 6 some conclusions are drawn regarding noise-induced effects in models of self-propelled motion. 


\section{Exact Quadrature Solution for the Diffusion Coefficient in the One-Dimensional Case}

The exact formula for the diffusion coefficient in the one-dimensional case $(d=1)$ from Ref. [11] (for the specific case $g(v)=\sqrt{D}$ ) reads

$$
D_{\text {eff }}=\frac{\int_{0}^{\infty} d v_{2} e^{U\left(v_{2}\right)}\left[\int_{v_{2}}^{\infty} d v_{1} e^{-U\left(v_{1}\right)} v_{1}\right]^{2}}{\left(D / m^{2}\right) \int_{0}^{\infty} d v_{3} e^{-U\left(v_{3}\right)}}
$$

where $U(v)=\gamma m v^{2 \alpha+2} /(2(\alpha+1) D)$. Inserting the potential, introducing new variables $u_{i}=v_{i}(\gamma m / D)^{1 /(2 \alpha+2)}$ and carrying out the integral in the denominator, one arrives at

$$
\begin{aligned}
D_{\text {eff }}= & \frac{D^{\frac{1-\alpha}{1+\alpha}} m^{\frac{2 \alpha}{1+\alpha}}}{\gamma^{\frac{2}{1+\alpha}}} \frac{(2 \alpha+2)^{\frac{2 \alpha+1}{2 \alpha+2}}}{\Gamma(1 /(2 \alpha+2))} \int_{0}^{\infty} d u_{2} \exp \left[\frac{u_{2}^{(2 \alpha+2)}}{2 \alpha+2}\right] \\
& \times\left\{\int_{u_{2}}^{\infty} d u_{1} \exp \left[-\frac{u_{1}^{(2 \alpha+2)}}{2 \alpha+2}\right] u_{1}\right\}^{2} .
\end{aligned}
$$

The first factor is the desired scaling law in $D$ and $\gamma$ while the remaining terms form a numerical factor that still depends on the exponent $\alpha$.

For $\alpha=0$ (normal Brownian motion) one recovers the simple relation

$$
D_{\text {eff }}=\gamma^{-2} D, \quad \alpha=0 .
$$

For $\alpha=1$ (friction force is proportional to the cube of speed) one has

$$
D_{\text {eff }}=\frac{m}{2 \gamma} \Gamma\left(\frac{3}{4}\right) \int_{0}^{\infty} d x \operatorname{erfc}^{2}\left(x^{2}\right) e^{x^{4}} \approx 0.4875 m / \gamma, \quad \alpha=1 .
$$

For higher $\alpha$, the double integral in (11) can be reduced to a single integral over a product of an exponential and an incomplete Gamma function. Here I just state the numerical factors resulting from the scaling law and the numerical evaluation of the remaining integral.

$$
\begin{array}{ll}
D_{\text {eff }} \approx 0.3737 m^{4 / 3} \gamma^{-2 / 3} D^{-1 / 3}, & \alpha=2 \\
D_{\text {eff }} \approx 0.3201 m^{3 / 2} \gamma^{-1 / 2} D^{-1 / 2}, & \alpha=3 .
\end{array}
$$

\section{Scaling of the Diffusion Coefficient in the Multi-Dimensional Case}

In the multi-dimensional case $(d>1)$, no analytical solution of the nonlinear diffusion problem given in (6) is known. However, as shown in this section, scaling relations can be derived by a simple transformation of space and time.

One may introduce new variables, making the system non-dimensional

$$
\tilde{x}_{1}=x_{1} / x_{0}, \quad \tilde{x}_{2}=x_{2} / x_{0}, \quad \tilde{x}_{3}=x_{3} / x_{0}, \quad \tilde{t}=t / t_{0} .
$$

This transforms the diffusion coefficient as follows

$$
\tilde{D}_{\text {eff }}=D_{\text {eff }} t_{0} / x_{0}^{2} .
$$


As shown in the following one can chose $x_{0}$ and $t_{0}$ as powers of the system's parameters such that in the resulting equations for $\tilde{x}$ and $\tilde{y}$ the noise intensity $D$, the friction coefficient $\gamma$, and the mass $m$ do not appear anymore. Then the left hand side of (17) does not depend on $D, \gamma$, nor $m$ and thus implies a scaling relation between $D_{\text {eff }}$ and $x_{0}, t_{0}$ which are powers of $D, \gamma$, and $m$.

Using the non-dimensional velocity $\tilde{\boldsymbol{v}}=\boldsymbol{v} t_{0} / x_{0}$, the stochastic equations read

$$
\dot{\tilde{\boldsymbol{x}}}=\tilde{\boldsymbol{v}}, \quad m \dot{\tilde{\boldsymbol{v}}}=-\gamma x_{0}^{2 \alpha} t_{0}^{1-2 \alpha} \tilde{\boldsymbol{v}}^{2 \alpha} \tilde{\boldsymbol{v}}+\sqrt{\frac{2 D t_{0}^{3}}{x_{0}^{2}}} \boldsymbol{\xi}(t) .
$$

I now assume that $t_{0}$ and $x_{0}$ depend like power laws on the diffusion, friction coefficients, and mass

$$
\begin{aligned}
t_{0} & =\hat{t}_{0}\left(D / D_{0}\right)^{\Delta_{1}}\left(\gamma / \gamma_{0}\right)^{\sigma_{1}}\left(m / m_{0}\right)^{\omega_{1}}, \\
x_{0} & =\hat{x}_{0}\left(D / D_{0}\right)^{\Delta_{2}}\left(\gamma / \gamma_{0}\right)^{\sigma_{2}}\left(m / m_{0}\right)^{\omega_{2}},
\end{aligned}
$$

where $\hat{t}_{0}, \hat{x}_{0}, D_{0}, \gamma_{0}$, and $m_{0}$ are arbitrary constants with physical dimensions of time, length, diffusion coefficient, friction coefficient, and mass respectively. The exponents $\Delta_{i}, \sigma_{i}, \omega_{i}$ (with $i=1,2$ ) are still to be determined by demanding that the resulting dynamics does not depend on $D, \gamma$, and $m$. Inserting the ansatz (19) one obtains

$$
\begin{aligned}
\dot{\tilde{\boldsymbol{x}}}= & \tilde{\boldsymbol{v}} \\
\dot{\tilde{\boldsymbol{v}}}= & -\hat{x}_{0}^{2 \alpha} \hat{t}_{0}^{1-2 \alpha} \frac{m^{2 \alpha \omega_{2}+(1-2 \alpha) \omega_{1}-1}}{m_{0}^{2 \alpha \omega_{2}+(1-2 \alpha) \omega_{1}}} \frac{\gamma^{2 \alpha \sigma_{2}+(1-2 \alpha) \sigma_{1}+1}}{\gamma_{0}^{2 \alpha \sigma_{2}+(1-2 \alpha) \sigma_{1}}} \frac{D^{2 \alpha \Delta_{2}+(1-2 \alpha) \Delta_{1}}}{D_{0}^{2 \alpha \Delta_{2}+(1-2 \alpha) \Delta_{1}}} \tilde{v}^{2 \alpha} \tilde{\boldsymbol{v}} \\
& +\sqrt{\frac{2 \hat{t}_{0}^{3}}{\hat{x}_{0}^{2}} \frac{\gamma^{3 \sigma_{1}-2 \sigma_{2}} m^{3 \omega_{1}-2 \omega_{2}-2} D^{3 \Delta_{1}-2 \Delta_{2}+1}}{\gamma_{0}^{3 \sigma_{1}-2 \sigma_{2}} m_{0}^{3 \omega_{1}-2 \omega_{2}} D_{0}^{3 \Delta_{1}-2 \Delta_{2}}}} \boldsymbol{\xi}(t),
\end{aligned}
$$

a dynamics which does not contain the noise intensity $D$ if

$$
1+3 \Delta_{1}-2 \Delta_{2}=0, \quad 2 \alpha \Delta_{2}+(1-2 \alpha) \Delta_{1}=0
$$

which is solved by

$$
\Delta_{1}=-\frac{\alpha}{1+\alpha}, \quad \Delta_{2}=\frac{1}{2} \frac{1-2 \alpha}{1+\alpha} .
$$

The exponents for the friction coefficient and mass can be determined in an analogous way by demanding that the prefactors of the friction term and the noise term do not depend on $\gamma$ and $m$ implying that the respective sums of exponents for each term in (20) vanish. Choosing the exponents in this way, the diffusion coefficient $\tilde{D}_{\text {eff }}$ of (20) does not depend on $m, \gamma$ and $D$ either; from (17) one can then infer that the diffusion coefficient of the original dynamics $D_{\text {eff }}$ scales like $x_{0}^{2} / t_{0}$. Using (19) together with the calculated exponents, one finally obtains

$$
D_{\mathrm{eff}} \sim m^{\frac{2 \alpha}{1+\alpha}} \gamma^{-\frac{2}{1+\alpha}} D^{\frac{1-\alpha}{1+\alpha}}
$$

This is again the same scaling law as in the one-dimensional case, cf. (11). The spatial dimension affects only the prefactor. Note, that unlike in the case of normal Brownian motion, 
the generalization to the multi-dimensional case is nontrivial because for $\alpha>0$ the dynamics of the velocity components is coupled through the speed $v$. For the same reason, I do not expect (as one could do for normal Brownian motion) that the diffusion coefficient is independent of the dimension $d$.

\section{Comparison to Simulation Results and Interpretation of the Findings}

In Fig. 1 I compare simulation results for the one-dimensional case to the theoretical findings. The power laws for $\alpha=1,2$, and 3 are in excellent agreement with the simulation results over three orders of magnitude. The scaling relations are also confirmed in the multidimensional cases, shown in Fig. 2; here the same slopes as in the one-dimensional case are found in a log-log plot.

Turning again to the one-dimensional case in Fig. 1, I note that for the specific values of friction coefficient and mass $(\gamma=1, m=1)$, it looks like all the curves intersect at one specific noise intensity around $D \approx 0.5$. For the one-dimensional case one can show that this is not strictly true: in the inset of Fig. 1 I show a blow-up of the region around the intersection points). Here simulations (symbols) over a large ensemble (necessary to increase the accuracy of the results) confirm the theoretical prediction (solid lines) that there is no unique intersection point for all the curves.

The region defined by the intersection points for $\alpha=0,1,2,3$ is even more narrow for the two- and three-dimensional cases shown in Fig. 2.

I have found that the results for the multi-dimensional case are well fit by the following relation

$$
D_{\mathrm{eff}} \approx(d+1)^{\frac{-2 \alpha}{1+\alpha}} m^{\frac{2 \alpha}{1+\alpha}} \gamma^{-\frac{2}{1+\alpha}} D^{\frac{1-\alpha}{1+\alpha}}
$$

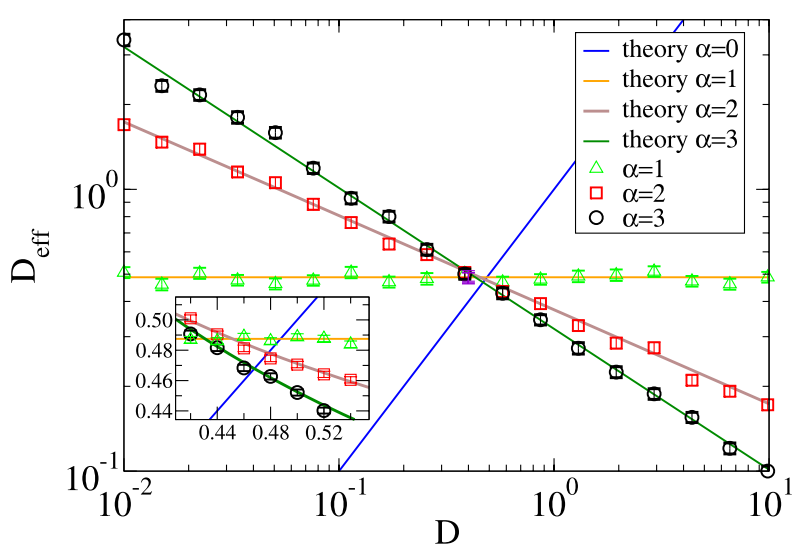

Fig. 1 Diffusion coefficient vs noise intensity $D$ for the one-dimensional case $(\gamma=1, m=1)$. The inset is a (linear scale) blow-up of the region around the intersection points showing indeed different intersection points for the family of curves which is also confirmed by three other sets of simulation results (symbols). Theory is according to (12) $(\alpha=0$, blue $),(13)(\alpha=1$, yellow), (14) $(\alpha=2$, brown), and (15) ( $\alpha=3$, green). In the simulation I used a simple stochastic Euler procedure (see e.g. [13], Sect. 3.6) with a time-step of $\Delta t=10^{-3}$ to simulate $10^{3}$ realizations. The mean square displacement of this ensemble at $t=10^{4}$ was used to estimate the diffusion coefficient. For the data shown in the inset I had to use more realizations $\left(N=10^{5}\right)$ and a shorter time $\left(t=10^{3}\right)$ in order to obtain reasonable accuracy in a feasible simulation time 

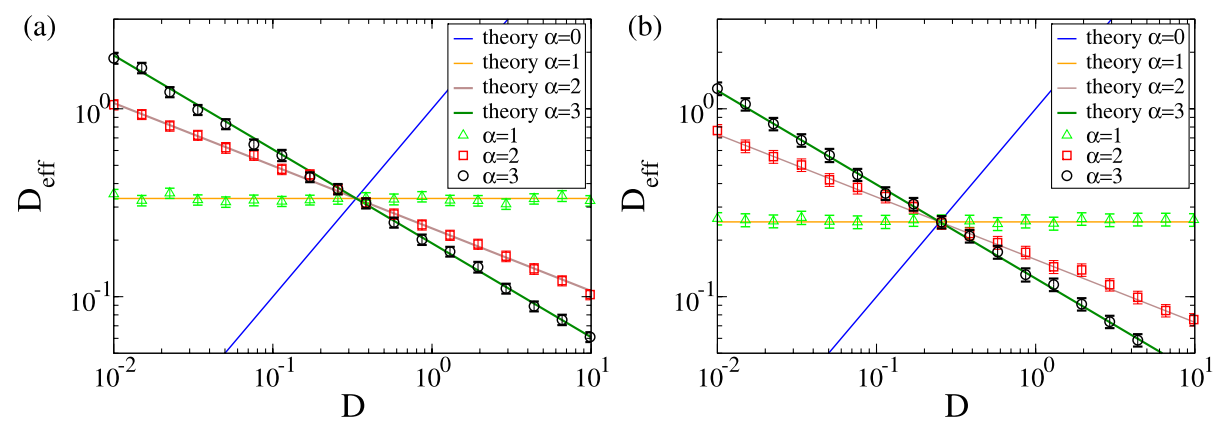

Fig. 2 Diffusion coefficient vs noise intensity $D$ for the two-dimensional (a) and three-dimensional (b) systems for $\gamma=1, m=1$. Solid lines are according to (24) with $d=2(\mathbf{a})$ and $d=3(\mathbf{b})$. The simulation algorithm and its parameters are the same as in Fig. 1

Note that according to this relation the intersection point for all $\alpha$ would be indeed one and the same: all the curves meet at $D^{*}=1 /(1+d)$ (which is certainly only an approximation). The relation (24) is shown in Fig. 2 by the different solid lines: it gives an excellent fit to all simulated data. For the one-dimensional case the agreement is still good (not shown) although small deviations are already visible by plotting the fit formula against the exact results from (13-15) in a log-log plot like Fig. 1. As an example take the curve for $\alpha=1$ : the exact results (13) predicts $D_{\text {eff }} \approx 0.4875$ whereas the fit formula (24) gives $D_{\text {eff }} \approx 0.5$, i.e., here the fit formula deviates by less than $3 \%$ from the exact result.

The fit formula (24) reduces for $\alpha=0$ to the well-known exact result

$$
D_{\text {eff }}=D / \gamma^{2}, \quad \alpha=0
$$

One may wonder how the dimensionality affects the diffusion coefficient. For normal Brownian motion the diffusion coefficient does not depend on the dimension at all (cf. (25)) because the mean square displacements in each spatial direction are independent and add up-so the mean square displacement in $d$ dimensions is the $d$-fold mean square displacement of the one-dimensional case and this is exactly the factor $d$ one divides by in the definition of the diffusion coefficient in (7). For a speed-dependent friction function, in contrast, the dynamics of the velocity components $v_{i}(i=1,2,3)$ is coupled through the speed and one cannot expect that the diffusion coefficient is independent of the system's spatial dimension. Taking (24) as an acceptable approximation, the spatial dimension enters the diffusion coefficient by a factor of

$$
(d+1)^{\frac{-2 \alpha}{1+\alpha}} \text {. }
$$

For $\alpha>0$ this factor is smaller than 1. Furthermore, the bigger $\alpha$ is the bigger is the correction to the case of normal Brownian motion.

The most remarkable result of this paper is also confirmed in all plots: for a cubic friction force, the diffusion coefficient does not depend at all on the noise intensity. Why is this so? How can for $\alpha>1$ the diffusion coefficient even drop with growing noise? For the discussion of this question, I restrict myself in what follows to the one-dimensional case.

The diffusion coefficient can be written as

$$
D_{\text {eff }}=\left\langle v^{2}\right\rangle \tau_{\text {corr }}
$$



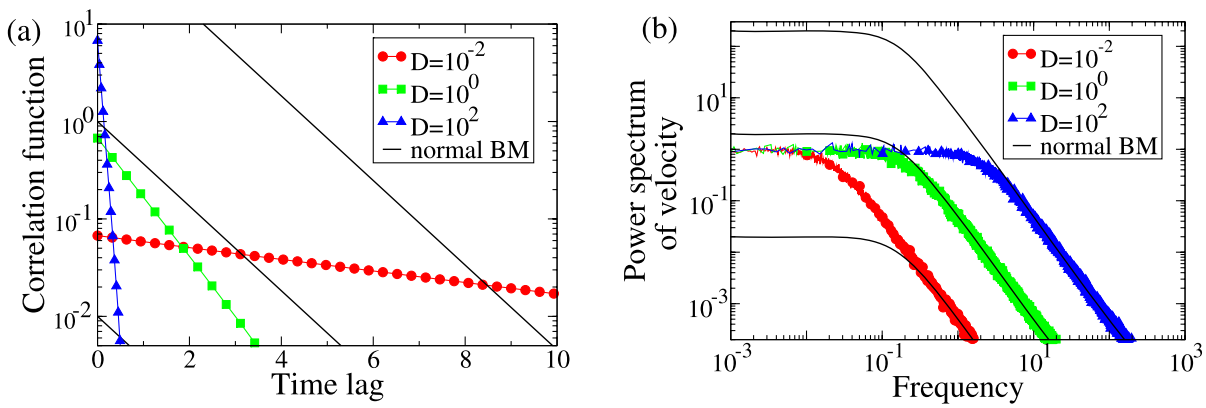

Fig. 3 Correlation functions (a) and power spectra (b) of the velocity for different values of the noise intensity, $m=1, \gamma=1, d=1$, and $\alpha=1$ (corresponding to a cubic friction law). In contrast to the case of normal Brownian motion (black solid lines), the slope changes in (a) with increasing noise indicating a change in the correlation time of the velocity. All the spectra in (b) start at the same value at zero frequency which equals twice the diffusion coefficient (cf. (28))

Here $\tau_{\text {corr }}=\int_{0}^{\infty} d \tau\langle v(t) v(t+\tau)\rangle /\left\langle v^{2}\right\rangle$ is the correlation time of the velocity and $\left\langle v^{2}\right\rangle$ is its variance. When increasing the noise intensity, one will typically increase the variance. Intuitively one associates this increase in the velocity's variance with a necessary increase in the spatial diffusion coefficient. However, the second factor becomes important for nonlinear friction. For Stokes friction $\tau_{\text {corr }}=\gamma^{-1}$, i.e. the correlation time does not depend on the noise intensity. For $\alpha=1$ the correlation time drops with increasing noise and in this way just balances the increase in variance such that the product (the diffusion coefficient) remains constant for all noise levels. The correlation time presumably drops with growing noise because larger noise implies larger values of the speed and also much stronger dissipation leading to a shorter memory of the process. With growing noise the velocity fluctuates stronger but also faster. In the opposite limit of very weak noise, typical values of the speed are small and thus dissipation is very weak. Here the velocity is a long correlated process and despite the fact that the variance approaches zero for vanishing noise, one still obtains a finite diffusion coefficient because the correlation time diverges in the same limit.

The above is confirmed quantitatively in Fig. 3a showing the correlation function in a lin-log plot: while the variance (the correlation function at the origin) increases with growing noise, the correlation itself decays the faster the stronger the noise is. This is evident in the lin-log plot since the decay seems to be very close to an exponential one and the decay rate (the slopes in the lin-log plot) increase strongly when increasing the noise. In the case of classical Brownian motion (solid lines) only the variance (the value of the correlation function at the origin) changes - the slope (the inverse correlation time) remains unchanged. I note that the constance of the diffusion coefficient implies that the integral over the correlation function is independent of the noise intensity. This integral is according to the Wiener-Khinchine theorem half of the power spectrum at zero frequency.

$$
\begin{aligned}
D_{\text {eff }} & =\int_{0}^{\infty} d \tau\langle v(t) v(t+\tau)\rangle \\
& =\left.\frac{1}{2} \int_{-\infty}^{\infty} d \tau\langle v(t) v(t+\tau)\rangle e^{i 2 \pi f \tau}\right|_{f=0}=\frac{1}{2} S(0) .
\end{aligned}
$$

For completeness I show in Fig. $3 \mathrm{~b}$ the entire spectrum as a function of frequency in the one-dimensional case for $\alpha=1$ and different values of the noise intensity. Indeed, the zerofrequency value is the same for all three curves. In contrast to that, for a normal Brownian 
motion, the zero-frequency level is proportional to the noise intensity. Remarkably, the highfrequency tail of the spectrum is $f^{2}$ and agrees with that of the normal Brownian motion. The Lorentz form of the spectrum corresponds to the exponential decay of the correlation function seen in Fig. 3a.

Now generalizing these findings for $\alpha>1$, one can conclude that with increasing noise the correlation time must decrease even faster than the variance increases resulting in a drop of the diffusion coefficient with growing noise. In the opposite limit of a vanishing noise intensity one obtains an extremely long-correlated velocity and hence a divergence of the diffusion coefficient.

\section{Two Generalizations of the Model}

Let me briefly consider two obvious extensions of the one-dimensional model that may be of interest.

First, I discuss what happens when one does not have a pure power law but the friction function starts at small speed at a finite positive value

$$
\gamma(v)=\gamma_{0}\left(\varepsilon+v^{2 \alpha}\right), \quad \varepsilon>0 .
$$

In this case, not much will change if typical velocities are high, i.e. at large noise. However, at weak and moderate noise, the system now experiences normal Stokes friction and will show the respective diffusion coefficient $D_{\text {eff }}=D /\left(\gamma_{0} \varepsilon\right)^{2}$. In result, one obtains a curve that starts with the diffusion law of normal Brownian motion $D_{\text {eff }} \sim D$ and then approaches the respective diffusion law for the pure power-law friction $D_{\text {eff }} \sim D^{(1-\alpha) /(1+\alpha)}$ discussed before. In particular, for $\alpha>1$ one can thus expect a maximum in the diffusion coefficient as a function of noise intensity.

Second, let me discuss what kind of diffusion is observed for an equilibrium systems with power law friction. An equilibrium model with nonlinear friction can be constructed by requiring that the noise intensity becomes speed dependent such that the generalized Einstein relation [12]

$$
D(v)=k_{B} T \gamma(v)
$$

is satisfied. I obtain in this case a Langevin equation with multiplicative white noise

$$
m \dot{v}=-\gamma(v) v+\sqrt{2 k_{B} T \gamma(v)} \xi(t)
$$

which is not uniquely determined because one still has to interpret the stochastic integral (Ito-Stratonovich dilemma, see, for instance, [14]). As pointed out by Klimontovich and others one has to choose the so-called kinetic interpretation in order to obtain a Maxwell distribution for the velocity; the more common Ito and Stratonovich interpretations will lead to different distributions. The Fokker-Planck equation (FPE) resulting from the kinetic interpretation $\partial_{t} P(v, t)=\partial_{v}\left[v \gamma(v) / m+\left(k_{B} T / m^{2}\right) \gamma(v) \partial_{v}\right] P(v, t)$ is indeed satisfied in steady-state $\left(\partial_{t} P(v, t)=0\right)$ by the Maxwell distribution $P_{0} \sim \exp \left[-m v^{2} /\left(2 k_{B} T\right)\right]$. In Ref. [11] the diffusion coefficient for such an equilibrium system has been found for the friction function

$$
\gamma(v)=\gamma_{0}\left(1+\beta v^{2}\right) .
$$


This function approaches the power law friction with $\alpha=1$ for $\beta=\gamma_{1} / \gamma_{0}$ and in the limit $\gamma_{0} \rightarrow 0$. The analytical result for the friction function (32) reads

$$
D_{\text {eff }}=\frac{k_{B} T}{\gamma_{0}} \sqrt{\pi} z e^{z^{2}} \operatorname{erfc}(z) \quad \text { with } z=\sqrt{\frac{\gamma_{0} m}{2 k_{B} T \gamma_{1}}}
$$

where $\operatorname{erfc}()$ is the complementary error function. Now taking the limit, one obtains

$$
\lim _{\gamma_{0} \rightarrow 0} D_{\text {eff }}=\infty
$$

a pure power-law friction in an equilibrium system obeying the generalized Einstein relation (30) and having a Maxwell velocity distribution leads to an infinite diffusion coefficient. This result can be understood as follows. In the equilibrium model (31), both drift and noise terms have a zero at $v=0$ if $\gamma(v)$ follows a power law. This prevents the trajectory from crossing zero-the dynamics is not ergodic anymore. The Maxwell distribution is still a steady-state solution of the FPE but corresponds to an ensemble average only if half of the initial velocities are chosen to be negative and the other half to be positive. Each of these sub-ensembles keeps the sign of its velocities and thus goes on average either to the right or left. This in turn implies a ballistic motion $\left(\left\langle x^{2}(t)\right\rangle \sim t^{2}\right)$ which is reflected in the divergence of the diffusion coefficient. This result depends strongly on whether one has really a pure power law-any small Stokes contribution will bring us back to (33) and thus to normal diffusion because then a crossing of the zero velocity point becomes possible again.

\section{Summary and Conclusions}

I have shown that the diffusion coefficient of a Brownian motion with a friction force depending on the speed like a power law, depends on the noise intensity like a power law as well. The dependence of the diffusion coefficient on the friction coefficient and on the particle's mass follows also power laws. The respective exponents have been determined for arbitrary spatial dimension. In the one-dimensional case the exact prefactor has been found. For the multi-dimensional case, I proposed a fit formula that works well as shown by comparison with stochastic simulations for $\alpha=1,2,3$, and $d=2,3$.

Only a few analytical results on diffusion with nonlinear friction (mostly for weak noise) are known $[6,7,11,15,16]$. Simple, yet nontrivial examples for nonlinear friction are those given by a power law. Furthermore, thinking of a Taylor expansion of a general friction function, we may also find in more involved models limit cases where only one of the powers is relevant. By the results derived in this paper one then knows the scaling of the diffusion coefficient in these limit cases, at least.

The results in this paper have also implications for models of self-propelled motion of biological objects. In Ref. [11] it has been shown that a model of active Brownian motion remarkably shows a minimum in the diffusion coefficient as a function of noise intensity. It is an interesting question whether the minimum of the diffusion coefficient vs noise intensity is a robust phenomenon or whether and to which extend it does depend on the specific model under investigation. As a matter of fact, the minimum relies on two properties of $\hat{\gamma}(v)$ which are sufficient (although not necessary): (i) it should be negative for small speeds $|v|<v_{0}$ leading to two stable finite velocities $\pm v_{0}$ for vanishing noise; (ii) the dominant behavior at very large velocities should be such that the diffusion coefficient grows with increasing noise. 
The latter condition is exactly the one clarified here in this paper. In Ref. [11] the simplified depot-model by Ebeling and coworkers $[4,9]$ was used which behaves at strong velocities like $\hat{\gamma}(v) \rightarrow \gamma_{0}$, i.e. for large speeds the model approaches Stokes friction (or in my notation $\alpha=0$ ) and hence for strong noise the diffusion coefficient grows like $D / \gamma_{0}^{2}$. Therefore, the simplified depot model meets condition (2) and thus shows the minimum vs noise intensity. However, another popular choice for a pumping/friction function is the Rayleigh model $\hat{\gamma}(v)=\left(v^{2}-v_{0}^{2}\right)$ which asymptotically behaves like a power law with $\alpha=1$ (i.e., a cubic friction force). Since for such a power law the diffusion coefficient does not depend on the noise intensity, the condition (ii) is not met and one cannot expect (though also not exclude) to observe a minimum in the diffusion coefficient.

In the introduction I noted that a Langevin equation with power-law friction may be interesting also as a potential colored-noise generator. When a colored noise drives a dynamical system the statistics of the noise can have a severe effect on the dynamics (see [17], discussing noise-induced currents in a ratchet system and its dependence on the noise statistics). The dynamics with $\alpha=1$ could be used to generate almost exponentially correlated noise with a smooth non-Gaussian statistics.

A number of open questions remain. The interesting case where $\alpha$ is negative has not been touched here-here it would be unclear whether the system shows normal diffusion at all. Furthermore, the interaction of a power-law friction and spatial force fields is another exciting and unexplored problem which will be investigated in the near future.

Acknowledgements I would like to thank Ernesto M. Nicola for inspiring discussions. I am furthermore indebted to Simon F. Nørrelykke for a number of very useful comments on an earlier version of this paper.

\section{References}

1. Brown, R.: A brief account of microscopical observations made in the months of June, July and August, 1827 , on the particles contained in the pollen of plants; and on the general existence of active molecules in organic and inorganic bodies. Philos. Mag. 4, 161 (1827)

2. Einstein, A.: Über die von der molekularkinetischen Theorie der Wärme geforderte Bewegung von in ruhenden Flüssigkeiten suspendierten Teilchen. Ann. Phys. (Leipz.) 17, 549 (1905)

3. Langevin, P.: Comptes Rendus 146, 530 (1908)

4. Schweitzer, F., Ebeling, W., Tilch, B.: Complex motion of Brownian particles with energy depots. Phys. Rev. Lett. 80, 5044 (1998)

5. Schienbein, M., Gruler, H.: Langevin equation, Fokker-Planck equation and cell migration. Bull. Math. Biol. 55, 585 (1993)

6. Mikhailov, A.S., Meinköhn, D.: Self-motion in physico-chemical systems far from thermal equilibrium. In: Schimansky-Geier, L., Pöschel, T. (eds.) Stochastic Dynamics, pp. 334. Springer, Berlin (1998)

7. Badoual, M., Jülicher, F., Prost, J.: Bidirectional cooperative motion of molecular motors. Proc. Natl. Acad. Sci. 99, 6696 (2002)

8. Erdmann, U., Ebeling, W., Schimansky-Geier, L., Ordemann, A., Moss, F.: Active Brownian particle and random walk theories of the motions of zooplankton: application to experiments with swarms of daphnia. http://arxiv.org/abs/q-bio.PE/0404018 (2004)

9. Erdmann, U., Ebeling, W., Schimansky-Geier, L., Schweitzer, F.: Brownian particles far from equilibrium. Eur. Phys. J. B 15, 105 (2000)

10. Hänggi, P., Jung, P.: Colored noise in dynamical systems. Adv. Chem. Phys. 89, 239 (1995)

11. Lindner, B.: The diffusion coefficient of nonlinear Brownian motion. New J. Phys. 9, 136 (2007)

12. Klimontovich, Y.L.: Statistical Theory of Open Systems. Kluwer Academic, Dordrecht (1995)

13. Risken, H.: The Fokker-Planck Equation. Springer, Berlin (1984)

14. Gardiner, C.W.: Handbook of Stochastic Methods. Springer, Berlin (1985)

15. Ebeling, W.: Nonlinear Brownian motion. Condens. Matter Phys. 7, 539 (2004)

16. Schimansky-Geier, L., Erdmann, U., Komin, N.: Advantages of hopping on a zig-zag course. Physica A 351, 51 (2005)

17. Doering, C.R., Horsthemke, W., Riordan, J.: Nonequilibrium fluctuation-induced transport. Phys. Rev. Lett. 72, 2984 (1994) 